\title{
Necessidade de módulo proteico para pacientes em estado grave: estudo das fórmulas enterais em sistema fechado disponíveis no mercado
}

\author{
Need of protein module for critically ill patients: study of enteral formulas in a closed system \\ available on the market
}

\section{DOI: $10.37111 /$ braspenj.2020352005}

José Henrique Silvah

Carolina Ferreira Nicoletti2

Cristiane Maria Mártires de Lima²

Arthur Welle ${ }^{3}$

Júlio Sérgio Marchini ${ }^{4}$

\section{Unitermos:}

Terapia Nutricional. Alimentos Formulados. Nutrição Enteral. Necessidade energética. Necessidade proteica.

\section{Keywords:}

Nutrition Therapy. Food, Formulated. Enteral Nutrition. Energy Requirement. Protein Requirement.

Endereço de correspondência:

José Henrique Silvah

Hospital das Clínicas de Ribeirão Preto, Departamento de Clínica Médica - Divisão de Nutrologia Avenida Bandeirantes, 3900 - Bairro Monte Alegre Ribeirão Preto, SP, Brasil - CEP: 14049-900

E-mail: ozeenrique@gmail.com

\section{Submissão}

26 de maio de 2020

Aceito para publicação

24 de junho de 2020

\begin{abstract}
RESUMO
Introdução: O suporte nutricional dos pacientes em estado grave deve ser individualizado e suprir as necessidades energéticas e proteicas diárias. Apesar das entidades de saúde publicarem suas respectivas recomendações de terapia nutricional para esses pacientes, orientações sobre o uso de módulos proteicos ainda são escassas e controversas. Este estudo objetivou avaliar a adequação proteico/energética das fórmulas enterais industrializadas existentes no mercado brasileiro utilizadas na terapia nutricional de pacientes adultos, assim como a necessidade de uso de módulos proteicos. Método: 46 fórmulas enterais encontradas no mercado brasileiro foram avaliadas, por meio de simulações matemáticas, em relação à adequação frente às recomendações proteicas, sem extrapolar a oferta energética (limite de $110 \%$ da necessidade diária). Para as simulações, compreendendo o módulo proteico, a oferta energética do módulo foi incluída na análise. Resultados: A razão proteínas/calorias variou entre 3,1 e 9,2. Para pacientes sem obesidade, somente a fórmula Peptamen Intense - Nestlé [razão proteína (g) $/ 100 \mathrm{kcal}=9,2$ ] atinge pelo menos $100 \%$ da necessidade proteica $(1,5$ a $2 \mathrm{~g}$ proteína $/ \mathrm{kg} / \mathrm{dia})$, com um volume calculado para 20 a $25 \mathrm{kcal} / \mathrm{kg}$. Para pacientes com IMC entre 30 e $36 \mathrm{~kg} / \mathrm{m}^{2}$, não há fórmula que atinja isoladamente a recomendação. A fórmula Peptamen Intense - Nestlé atinge as recomendações para pacientes com IMC entre 36 e 40 e IMC $>44 \mathrm{~kg} / \mathrm{m}^{2}$. A comparação da fórmula Peptamen Intense - Nestlé com o restante das fórmulas disponíveis no mercado acrescidas de módulo proteico mostra que a Fresubin $2 \mathrm{kcal} \mathrm{HP}$ - Fresenius necessita do menor volume do conjunto (fórmula enteral + módulo proteico) para atingir $1000 \mathrm{kcal} \mathrm{e} 92 \mathrm{~g}$ de proteína $(25,5 \%$ menor que a Peptamen Intense - Nestlé). Conclusão: A maioria das fórmulas enterais disponíveis no mercado brasileiro não é capaz de ofertar, isoladamente, um aporte proteico adequado, sem gerar excesso de oferta energética (overfeeding). $O$ uso de módulos proteicos pode trazer benefícios frente à recuperação dos pacientes, inclusive os infectados pela COVID-19.
\end{abstract}

\section{ABSTRACT}

Introduction: The nutritional support of critically ill patients must be individualized and meet the daily energy and protein needs. Despite the fact that health entities publish their respective nutritional therapy recommendations for these patients, guidelines on the use of protein modules are still scarce and controversial. This study aimed to evaluate the protein / energy adequacy of the industrialized enteral formulas existing in the Brazilian market used in the nutritional therapy of adult patients, as well as the need to use protein modules. Methods: 46 enteral formulas found in the Brazilian market were evaluated, by means of mathematical simulations, in relation to the adequacy against protein recommendations, without extrapolating the energy supply (limit of $110 \%$ of the daily requirement). For the simulations, comprising the protein module, the energy supply of the module was included in the analysis. Results: The protein / calorie ratio varied between 3.1 and 9.2. For patients without obesity, only the Peptamen Intense - Nestlé formula [protein ratio $(\mathrm{g}) / 100 \mathrm{kcal}=9.2$ ] reaches at least $100 \%$ of the protein requirement $(1.5$ to $2 \mathrm{~g}$ protein $/ \mathrm{kg} / \mathrm{day})$, with a volume calculated to 20 to $25 \mathrm{kcal} / \mathrm{kg}$. For patients with BMI between 30 and $36 \mathrm{~kg} / \mathrm{m}^{2}$, there is no formula that achieves the recommendation in isolation. The Peptamen Intense - Nestle formula meets the recommendations for patients with a BMI between 36 and 40 and a BMl> $44 \mathrm{~kg} / \mathrm{m}^{2}$. The comparison of the Peptamen Intense - Nestlé formula with the rest of the formulas available on the market plus protein module shows that Fresubin $2 \mathrm{kcal}$ HP - Fresenius needs the lowest volume of the set (enteral formula + protein module) to reach 1000 $\mathrm{kcal}$ and $92 \mathrm{~g}$ of protein (25.5\% less than Peptamen Intense - Nestlé). Conclusion: Most of the enteral formulas available in the Brazilian market are not able to offer, in isolation, an adequate protein supply, without generating excess energy supply (overfeeding). The use of protein modules can bring benefits to the recovery of patients, including those infected by COVID-19.

1. Doutor em Ciências Médicas pela Faculdade de Medicina de Ribeirão Preto - Universidade de São Paulo (FMRP-USP), Ribeirão Preto, SP, Brasil. 2. Doutora em Ciências Médicas pela Faculdade de Medicina de Ribeirão Preto - Universidade de São Paulo (FMRP-USP), Ribeirão Preto, SP, Brasil. Mestre em Relações Sociais pela Universidade Estadual de Campinas (UNICAMP), Ribeirão Preto, SP, Brasil.

4. Professor Doutor da Divisão de Nutrologia do Departamento de Clínica Médica da Faculdade de Medicina de Ribeirão Preto - Universidade de São Paulo (FMRP-USP), Ribeirão Preto, SP, Brasil. 


\section{INTRODUÇÃO}

O suporte nutricional dos pacientes graves, especialmente em relação às necessidades calóricas e proteicas, tem sido alvo de constante debate. Atualmente, com a pandemia da COVID-19 (causada pelo vírus SARS-CoV-2), foi questionado se seria adequado o uso de módulos proteicos, devido ao risco de exposição e contágio pela manipulação dos mesmos. Deve ser enfatizado que o suporte nutricional de pacientes graves em unidades de terapia intensiva (UTI) é semelhante nos pacientes com e sem COVID-19', incluindo naqueles com restrição hídrica na presença de disfunção respiratória ou renal ${ }^{2}$.

Está bem descrito na literatura que a longa permanência em UTIs está diretamente associada ao pior prognóstico nutricional e à incidência de subnutrição, perda de massa muscular e funcionalidade, o que se associa a pior qualidade de vida e morbidade adicional ${ }^{3}$. Assim, recomenda-se que todos os pacientes recebam assistência nutricional individualizada com base na evolução clínica diária, visando suprir as necessidades diárias de energia, proteína e micronutrientes ${ }^{3-6}$. Estratégias para provisão da recomendação proteica devem ser enfatizadas, uma vez que trabalhos prévios demonstraram que pacientes críticos recebem pouca proteína, em média 0,7 g/ kg/ $\mathrm{d}^{7,8}$, e mais raramente acima de $1,2 \mathrm{~g} / \mathrm{kg}^{6}$, embora a ingestão proteica adequada seja possivelmente mais importante para redução de mortalidade que a energética?.

Recentemente, entidades de saúde levantaram novamente esse assunto ao publicarem suas respectivas recomendações para o suporte nutricional de pacientes com COVID-19 (Tabela 1). Dentre as orientações, sugere-se o uso de fórmulas enterais com alta densidade calórica (entre 1,5 a $2 \mathrm{kcal} / \mathrm{mL}$ ) para pacientes com restrição de fluidos ${ }^{2}$. Entretanto, as recomendações sobre o uso de módulos proteicos para pacientes graves, inclusive com COVID-19, ainda são controversas. Assim, esse estudo teve como objetivo avaliar a adequação proteico/energética das fórmulas enterais industrializadas existentes no mercado brasileiro utilizadas na terapia nutricional de pacientes adultos graves, assim como a necessidade de uso de módulos proteicos.

\section{MÉTODO}

Em abril de 2020, fórmulas enterais em sistema fechado encontradas no mercado brasileiro foram avaliadas em simulações matemáticas quanto à entrega de 100\% da necessidade proteica diária recomendada, sem extrapolar uma oferta calórica além de $110 \%$ da necessidade energética diária. A composição das diferentes fórmulas enterais foi obtida por meio do levantamento de informações dos portfólios, guias de produtos impressos ou por informações disponibilizadas online pelos fabricantes. Suplementos nutricionais orais não foram incluídos na análise.

As necessidades energéticas foram calculadas como a seguir ${ }^{1,2,4,5}$ :

- 20 a $25 \mathrm{kcal} / \mathrm{kg}$ de peso atual para pacientes sem obesidade;
Tabela 1 - Recomendações energéticas e proteicas para pacientes com COVID-19.

\begin{tabular}{|c|c|c|c|}
\hline Entidade & $\begin{array}{c}\text { Oferta energética } \\
\text { diária }\end{array}$ & $\begin{array}{l}\text { Oferta proteica } \\
\text { diária }\end{array}$ & $\begin{array}{c}\text { Fórmula } \\
\text { enteral }\end{array}$ \\
\hline ESPEN ${ }^{\star 3}$ & $\begin{array}{c}27 \text { kcal/kg (30 } \\
\text { kcal/kg para } \\
\text { gravemente } \\
\text { desnutridos, de } \\
\text { acordo com a } \\
\text { condição clínica e } \\
\text { tolerância) }\end{array}$ & $\begin{array}{c}1 \mathrm{~g} / \mathrm{kg} \text { em pa- } \\
\text { cientes idosos } \\
\text { ( } \geq 1 \mathrm{~g} / \mathrm{kg} \text { para } \\
\text { pacientes } \\
\text { polimórbidos) }\end{array}$ & $\begin{array}{c}\text { Sem } \\
\text { informações }\end{array}$ \\
\hline ASPEN $^{1}$ & $\begin{array}{c}15 \text { a } 20 \mathrm{kcal} / \mathrm{kg} \\
\text { de peso atual na } \\
1^{\text {a }} \text { semana }\end{array}$ & $\begin{array}{c}1,2 \text { a } 2 \mathrm{~g} / \mathrm{kg} \text { de } \\
\text { peso atual na } 1^{\mathrm{a}} \\
\text { semana } \\
1,2 \text { a } 2 \mathrm{~g} / \mathrm{kg} \text { de } \\
\text { peso atual na } 1^{\mathrm{a}} \\
\text { semana }\end{array}$ & $\begin{array}{l}\text { Padrão polimé- } \\
\text { rica, isosmótica, } \\
\text { com } \geq 20 \% \text { em } \\
\text { proteínas. } \\
\text { Módulo proteico } \\
\text { se necessário }\end{array}$ \\
\hline $\begin{array}{l}\text { BRASPEN/ } \\
\text { AMIB }^{2}\end{array}$ & $\begin{array}{c}\text { Iniciar com } 15 \text { a } \\
20 \mathrm{kcal} / \mathrm{kg} ; 25 \\
\mathrm{kcal} / \mathrm{kg} \text { após o } \\
4^{\circ} \text { dia }\end{array}$ & $\begin{array}{c}1,5 \text { a } 2 \mathrm{~g} / \mathrm{kg}, \\
\text { mesmo em caso } \\
\text { de disfunção } \\
\text { renal } \\
\text { Gradativa: } \\
0,8 \mathrm{~g} / \mathrm{kg} \text { nos } \\
\text { primeiros } 2 \text { dias, } \\
0,8 \text { a } 1,2 \text { nos } \\
\text { dias } 3 \text { a } 5 ;>1,2 \text { a } \\
\text { partir do } 5^{\circ} \text { dia }\end{array}$ & $\begin{array}{c}1,5 \text { a } 2 \text { kcal/mL } \\
\text { em pacientes } \\
\text { com restrição } \\
\text { hídrica. } \\
\text { Evitar uso de } \\
\text { módulo proteico } \\
\text { (risco de } \\
\text { contaminação } \\
\text { da enfermagem } \\
\text { por maior } \\
\text { manipulação) }\end{array}$ \\
\hline $\mathrm{AMIB}^{4}$ & $\begin{array}{c}\text { Iniciar com } 15 \\
\text { a } 20 \mathrm{kcal} / \mathrm{kg} ; \\
\text { Progredir para } \\
25 \mathrm{kcal} / \mathrm{kg} \mathrm{na} 1^{\mathrm{a}} \\
\text { semana } \\
\text { Iniciar com } 15 \mathrm{a} \\
20 \mathrm{kcal} / \mathrm{kg} \\
\text { Progredir para } \\
25 \mathrm{kcal} / \mathrm{kg} \text { na } 1^{\mathrm{a}} \\
\text { semana } \\
\end{array}$ & $\begin{array}{c}1,3 \text { a } 2 \mathrm{~g} / \mathrm{kg} \\
\text { Gradativa: } 0,8 \mathrm{~g} / \\
\text { kg nos primeiros } \\
2 \text { dias; } 0,8 \text { a } 1,2 \\
\mathrm{~g} / \mathrm{kg} \text { nos dias } 3 \\
\text { a } 5 ;>1,2 \mathrm{~g} / \mathrm{kg} \text { a } \\
\text { partir do } 5^{\circ} \text { dia }\end{array}$ & $\begin{array}{c}\text { Sem contra- } \\
\text { indicação a } \\
\text { módulos } \\
\text { proteicos }\end{array}$ \\
\hline $\begin{array}{l}\text { ASPEN / AMIB } \\
\text { pacientes com } \\
\text { obesidade }^{4,5}\end{array}$ & $\begin{array}{c}\text { IMC: } 30-50 \\
(11-14 \mathrm{kcal} / \mathrm{kg} \\
\text { peso atual), IMC } \\
>50 \text { (22-25 kcal/ } \\
\text { kg peso ideal) }\end{array}$ & $\begin{array}{l}\text { IMC: } 30-40 \text { (2 g/ } \\
\text { kg peso ideal); } \\
\text { IMC }>40 \text { ( } 2,5 \text { g/ } \\
\text { kg peso ideal) }\end{array}$ & $\begin{array}{c}\text { Sem contra- } \\
\text { indicação a } \\
\text { módulos } \\
\text { proteicos }\end{array}$ \\
\hline
\end{tabular}

$\overline{\mathrm{AMIB}}$ = Associação de Medicina Intensiva Brasileira; ASPEN = American Society of Parenteral and Enteral Nutrition; BRASPEN (SBNEP) = Sociedade Brasileira de Nutrição Enteral e Parenteral; ESPEN = European Society of Parenteral and Enteral Nutrition ( ${ }^{*}$ Recomendações para pacientes de enfermaria, polimórbidos e/ou geriátricos).

- $14 \mathrm{kcal} / \mathrm{kg}$ de peso atual para pacientes com índice de massa corporal (IMC) entre 30 e $50 \mathrm{~kg} / \mathrm{m}^{2}$;

- $25 \mathrm{kcal} / \mathrm{kg}$ de peso ideal para pacientes com IMC > 50 $\mathrm{kg} / \mathrm{m}^{2}$.

A necessidade proteica foi calculada como a seguir:

- 1,5 a $2 \mathrm{~g} / \mathrm{kg}$ de peso atual para pacientes sem obesidade;

- $2 \mathrm{~g} / \mathrm{kg}$ de peso ideal máximo para pacientes com IMC entre 30 e $40 \mathrm{~kg} / \mathrm{m}^{2}$;

- $2,5 \mathrm{~g} / \mathrm{kg}$ de peso ideal máximo para pacientes com IMC $>40 \mathrm{~kg} / \mathrm{m}^{2}$. 
Inicialmente, todas as fórmulas foram avaliadas nas quatro situações a seguir:

- pacientes sem obesidade com peso entre 50 e $100 \mathrm{~kg}$;

- com IMC entre 30 e $40 \mathrm{~kg} / \mathrm{m}^{2}$;

- com IMC entre 40 e $50 \mathrm{~kg} / \mathrm{m}^{2}$;

- com IMC > $50 \mathrm{~kg} / \mathrm{m}^{2}$.

Na sequência, a fórmula B-1, isoladamente, com 1,0 kcal/ $\mathrm{ml} \mathrm{e} \mathrm{9,2} \mathrm{g} \mathrm{de} \mathrm{proteína/100} \mathrm{mL} \mathrm{(9,2} \mathrm{g} \mathrm{proteína/100} \mathrm{kcal),} \mathrm{foi}$ comparada a todas as outras fórmulas disponíveis somadas a módulo proteico, para que se atingisse a mesma infusão de calorias e proteína (1000 kcal e $92 \mathrm{~g}$ de proteína). Para cada 15 gramas de módulo proteico considerou-se um volume de $100 \mathrm{~mL}$. Para as simulações incluindo módulo proteico, a oferta energética do módulo foi incluída na análise. A adequação das fórmulas enterais em relação às necessidades energético-proteicas, com e sem módulo proteico associado, foi apresentada sob a forma de figuras. A razão proteína (g)/100 kcal foi usada para classificar as fórmulas com maior aplicabilidade.

\section{RESULTADOS}

\section{Caracterização das Fórmulas Disponíveis no Mercado}

Um total de 46 fórmulas enterais de 5 indústrias diferentes (Nestlé, Fresenius, Danone/Nutricia, Abbott e BBraun) foram avaliadas em relação à densidade energética, oferta proteica e razão proteína (g)/100 kcal (Tabela 2). As indústrias Abbott, Nestlé, Fresenius, Danone/Nutricia e BBraun apresentam em seus respectivos portfólios, 40\%, 50\%, 41,7\%, 30\% e 33,3\% de fórmulas com densidade calórica $>1,5 \mathrm{kcal} / \mathrm{mL}$. A razão proteína (g)/100 kcal variou entre 4,2 a 5 (Abbott), 3,6 a 9,2 (Nestlé), 3,1 a 6,7 (Fresenius), 4 a 5,9 (Danone/Nutricia) e 3,8 a 5 (BBraun).

\section{Pacientes sem Obesidade}

A Figura 1 demonstra a adequação das fórmulas avaliadas em relação às necessidades estimadas de proteínas. Em um volume necessário para $20 \mathrm{kcal} / \mathrm{kg}$, a fórmula Peptamen Intense - Nestlé oferta $2 \mathrm{~g}$ proteína $/ \mathrm{kg} / \mathrm{dia}$ e Fresubin Lipid próximo de $1,5 \mathrm{~g}$ proteína $/ \mathrm{kg} / \mathrm{dia}$. Em um volume para $25 \mathrm{kcal} / \mathrm{kg}$, a fórmula Peptamen Intense - Nestlé atinge $2 \mathrm{~g}$ proteína $/ \mathrm{kg} / \mathrm{dia}$ e as seguintes fórmulas entregam pelo menos $1,5 \mathrm{~g}$ proteína/ kg/dia: Impact - Nestlé, Impact 1.5 - Nestlé, Novasource Senior - Nestlé, Novasource GC HP - Nestlé, Novasource Prol - Nestlé, Recovan - Fresenius, Fresubin Lipid - Fresenius, Nutrison Advanced Cubison - Danone/Nutricia e Nutrison Advanced Protison - Danone/Nutricia (todas com 5,2 g ou mais de proteína/100 kcal). Em relação às fórmulas com densidade energética $\geq 1,5 \mathrm{kcal} / \mathrm{mL}$, as que atingem 1,5 gramas de proteína $/ \mathrm{kg} /$ dia: Fresubin Liquid - Fresenius (com volume para ofertar $25 \mathrm{kcal} / \mathrm{kg}$ ) e Impact - Nestlé (com volume para ofertar $25 \mathrm{kcal} / \mathrm{kg}$ ), fórmulas com 6,2 g ou mais de proteína $/ 100 \mathrm{kcal}$. Somente uma fórmula Peptamen Intense - Nestlé com 9,2 g proteína/100 kcal atinge a adequação em todas as situações.
Tabela 2 - Caracterização das 46 fórmulas enterais disponíveis no mercado.

\begin{tabular}{|c|c|c|c|c|}
\hline Indústria & Fórmula & $\begin{array}{c}\text { Densidade } \\
\text { energética } \\
\text { (kcal/mL) }\end{array}$ & $\begin{array}{c}\text { Oferta } \\
\text { proteica } \\
\text { (g/ } 100 \\
\mathrm{~mL})\end{array}$ & $\begin{array}{c}\text { Proteína } \\
\text { (g)/100 } \\
\text { kcal }\end{array}$ \\
\hline \multirow{5}{*}{ Abbott } & Osmolite HN & 1,2 & 5,6 & 4,7 \\
\hline & Jevity Plus & 1,2 & 5,6 & 4,7 \\
\hline & Jevity 1.5 & 1,5 & 6,4 & 4,3 \\
\hline & Glucerna 1.0 & 1,0 & 4,2 & 4,2 \\
\hline & Glucerna 1.5 & 1,5 & 7,5 & 5,0 \\
\hline \multirow{16}{*}{ Nestlé } & Peptamen Intense & 1,0 & 9,2 & 9,2 \\
\hline & Peptamen Prebio & 1,0 & 4,0 & 4,0 \\
\hline & Peptamen 1.5 & 1,5 & 6,8 & 4,4 \\
\hline & Peptamen HN & 1,3 & 6,6 & 5,0 \\
\hline & Novasource hi protein & 1,5 & 7,7 & 5,1 \\
\hline & Novasource Ren & 2,0 & 7,4 & 3,7 \\
\hline & Impact & 1,1 & 6,5 & 6,1 \\
\hline & Impact 1.5 & 1,5 & 9,4 & 6,2 \\
\hline & Novasource Gl control & 1,5 & 6,0 & 4,0 \\
\hline & Isosource Mix & 1,2 & 4,3 & 3,6 \\
\hline & Isosource 1.5 & 1,5 & 6,3 & 4,2 \\
\hline & Novasource Senior & 1,2 & 6,5 & 5,2 \\
\hline & Novasource GC & 1,1 & 4,9 & 4,5 \\
\hline & Novasource GC 1.5 & 1,5 & 7,5 & 5,0 \\
\hline & Novasource GC HP & 1,1 & 6,4 & 5,8 \\
\hline & Novasource Proline & 1,0 & 5,8 & 5,8 \\
\hline \multirow{12}{*}{ Fresenius } & Fresubin Original & 1,0 & 3,8 & 3,8 \\
\hline & Fresubin Soya Fibre & 1,0 & 3,8 & 3,8 \\
\hline & Fresubin 1.2 HP Fibre & 1,2 & 6,0 & 5,0 \\
\hline & Fresubin Energy & 1,5 & 5,6 & 6,7 \\
\hline & Fresubin HP Energy & 1,5 & 7,5 & 5,0 \\
\hline & Fresubin 2kcal HP & 2,0 & 10 & 5,0 \\
\hline & Diben & 1,0 & 4,7 & 4,7 \\
\hline & Diben $1.5 \mathrm{HP}$ & 1,5 & 7,5 & 5,0 \\
\hline & Reconvan & 1,0 & 5,5 & 5,5 \\
\hline & Survimed OPD & 1,0 & 4,5 & 4,5 \\
\hline & Fresubin Hepa & 1,3 & 4,0 & 3,1 \\
\hline & Fresubin Lipid & 1,5 & 10 & 6,7 \\
\hline \multirow{10}{*}{$\begin{array}{l}\text { Danone/ } \\
\text { Nutricia }\end{array}$} & Nutrison & 1,0 & 4,0 & 4,0 \\
\hline & Nutrison Advanced Cubison & 1,0 & 5,5 & 5,5 \\
\hline & Nutrison Advanced Diason & 1,0 & 4,3 & 4,3 \\
\hline & $\begin{array}{c}\text { Nutrison Advanced Diason } \\
\text { Energy HP }\end{array}$ & 1,5 & 7,7 & 5,1 \\
\hline & Nutrison Advanced Peptisorb & 1,0 & 4,0 & 4,0 \\
\hline & Nutrison Advanced Protison & 1,3 & 7,5 & 5,9 \\
\hline & Nutrison Energy & 1,5 & 6,0 & 4,0 \\
\hline & Nutrison Multifiber & 1,0 & 4,0 & 4,0 \\
\hline & Nutrison Protein Plus Energy & 1,5 & 7,5 & 5,0 \\
\hline & Nutrison Protein Plus MF & 1,25 & 6,3 & 5,0 \\
\hline \multirow{3}{*}{ BBraun } & Nutricomp Standard & 1,0 & 3,8 & 3,8 \\
\hline & Nutricomp Energy HN & 1,5 & 7,5 & 5,0 \\
\hline & Nutricomp D & 1,0 & 4,1 & 4,1 \\
\hline
\end{tabular}




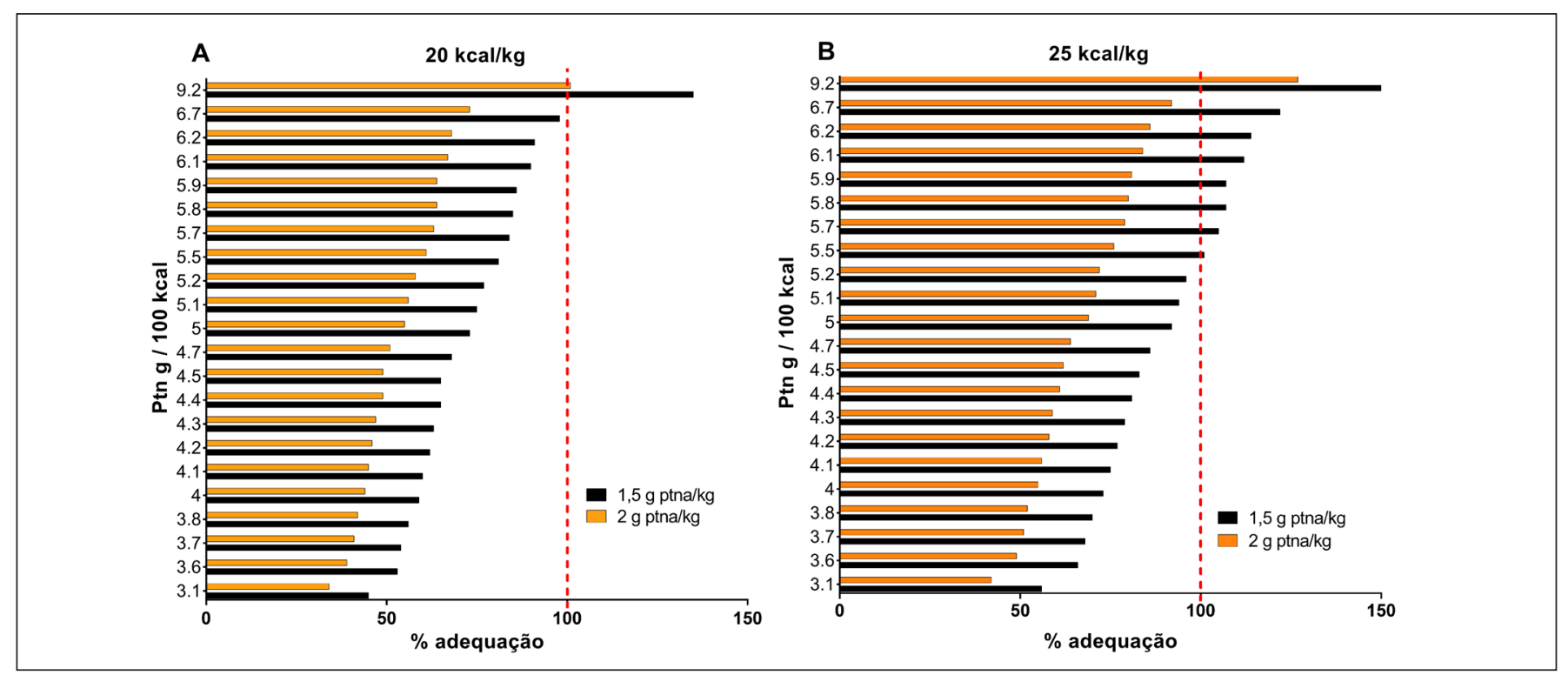

Figura 1 - Adequação (\%) da oferta proteica das fórmulas enterais avaliadas no presente estudo frente às recomendações para pacientes não obesos de acordo com a razão proteína/kcal. Utilizou-se para o cálculo a recomendação de $20 \mathrm{kcal} / \mathrm{kg}$ e 1,5 g/kg ou $2 \mathrm{~g} / \mathrm{kg}$ (A) ou $25 \mathrm{kcal} / \mathrm{kg}$ e $1,5 \mathrm{~g} / \mathrm{kg}$ ou $2 \mathrm{~g} / \mathrm{kg}$ (B), considerando indivíduo com IMC $=22,5 \mathrm{~kg} / \mathrm{m}^{2}$. O marco $100 \%$ indica se a fórmula enteral atinge a recomendação em sua totalidade.

\section{Pacientes com Obesidade}

A Figura 2 demonstra a adequação das fórmulas avaliadas em relação às necessidades estimadas (calorias e proteínas). A fórmula Peptamen Intense - Nestlé atinge 2 gramas de proteína $/ \mathrm{kg} /$ dia com volume ajustado para $14 \mathrm{kcal} / \mathrm{kg}$ de peso atual, para pacientes com IMC entre 36 e $40 \mathrm{~kg} / \mathrm{m}^{2}$. Para pacientes com IMC entre 30 e $36 \mathrm{~kg} / \mathrm{m}^{2}$, não há fórmula que atinja a recomendação. Para pacientes com IMC a partir de $44 \mathrm{~kg} / \mathrm{m}^{2}$, a fórmula Peptamen Intense - Nestlé atinge 2,5 gramas de proteína $/ \mathrm{kg} /$ dia com volume ajustado para $14 \mathrm{kcal} /$ $\mathrm{kg}$ de peso atual (recomendação para IMC entre 40 e $50 \mathrm{~kg} /$ $\mathrm{m}^{2}$ ), bem como para um volume ajustado para $25 \mathrm{kcal} / \mathrm{kg}$ de peso ideal (recomendação para $I M C>50 \mathrm{~kg} / \mathrm{m}^{2}$ ). Não há fórmulas com densidade energética $\geq 1,5 \mathrm{kcal} / \mathrm{mL}$ que atinjam a recomendação proteica para pacientes com obesidade.

\section{Análise Comparativa do Volume para Equivalência Energético-Proteica}

A fórmula Peptamen Intense - Nestlé, única que isoladamente atinge as necessidades em quase todas as situações, foi comparada ao restante das fórmulas disponíveis no mercado acrescidas de módulo proteico, em relação ao volume necessário para a equivalência energético-proteica (Figura 3).

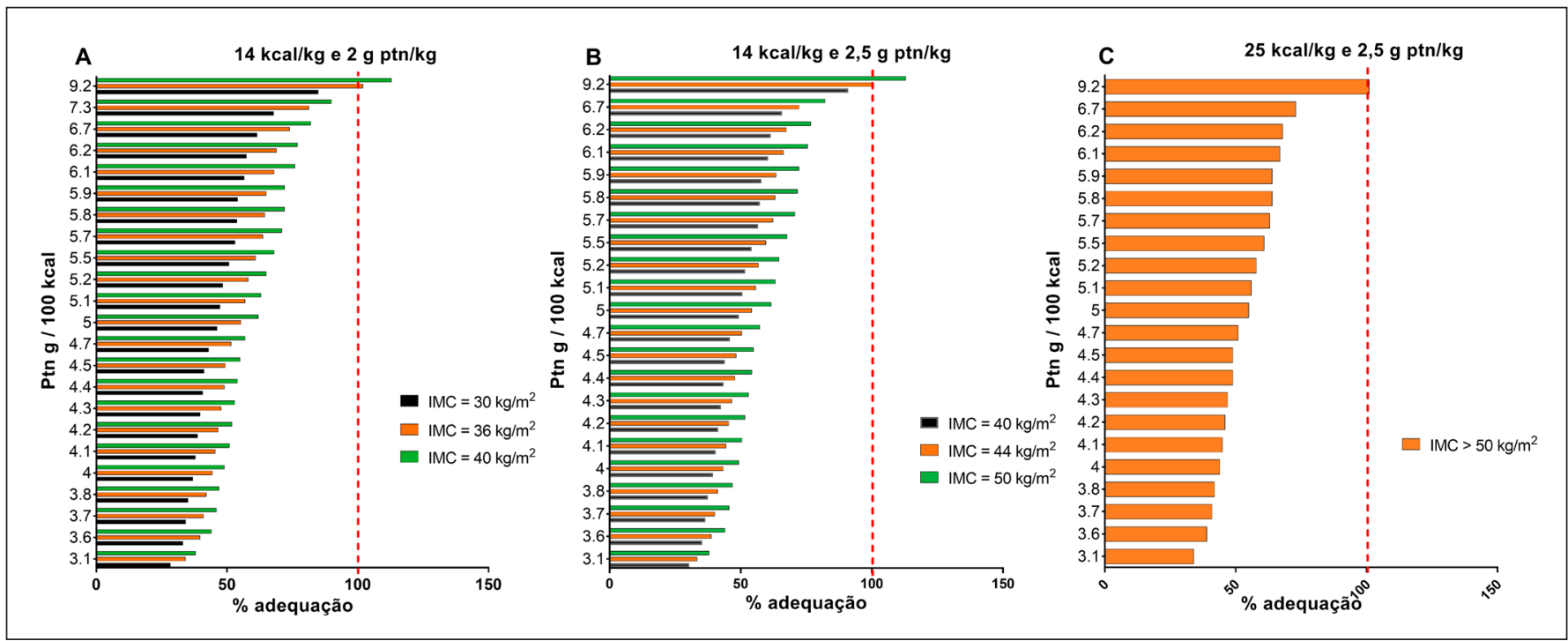

Figura 2 - Adequação (\%) da oferta proteica das fórmulas enterais avaliadas no presente estudo frente às recomendações para pacientes com obesidade de acordo com a razão proteína/kcal. Utilizou-se para o cálculo a recomendação de $14 \mathrm{kcal} / \mathrm{kg}$ e $2 \mathrm{~g} / \mathrm{kg}$ para indivíduos com IMC entre 30 e $40 \mathrm{~kg} / \mathrm{m}^{2}$ (A), ou $14 \mathrm{kcal} / \mathrm{kg}$ e $2,5 \mathrm{~g} / \mathrm{kg}$ para indivíduos com IMC entre 40 e $50 \mathrm{~kg} / \mathrm{m}^{2}$ (B) ou $25 \mathrm{kcal} / \mathrm{kg}$ e 2,5 g/kg para indivíduos com IMC maior que $50 \mathrm{~kg} / \mathrm{m}^{2}$ (C). O marco $100 \%$ indica se a fórmula enteral atinge a recomendação em sua totalidade. 
O menor volume do conjunto (fórmula enteral + módulo proteico) para atingir $1000 \mathrm{kcal}$ e $92 \mathrm{~g}$ de proteína foi verificado com a Fresubin $2 \mathrm{kcal} \mathrm{HP}$ - Fresenius (fórmula $=$ $395 \mathrm{~mL}+$ módulo $=350 \mathrm{~mL}$; volume total $=745 \mathrm{~mL}$ ), $25,5 \%$ menor que a Peptamen Intense - Nestlé (menos $255 \mathrm{~mL}$ ). Por outro lado, as fórmulas Fresubin Original
- Fresenius, Fresubin Soya Fibre - Fresenius e Nutricomp Standard - BBraun são as que resultam em maior volume (1 $170 \mathrm{~mL}$ ou $17 \%$ maior que a Peptamen Intense - Nestlé). Como esperado, fórmulas com maior densidade energética resultam em menor volume total para a equivalência energético-proteica (Figuras 4 a 7 ).

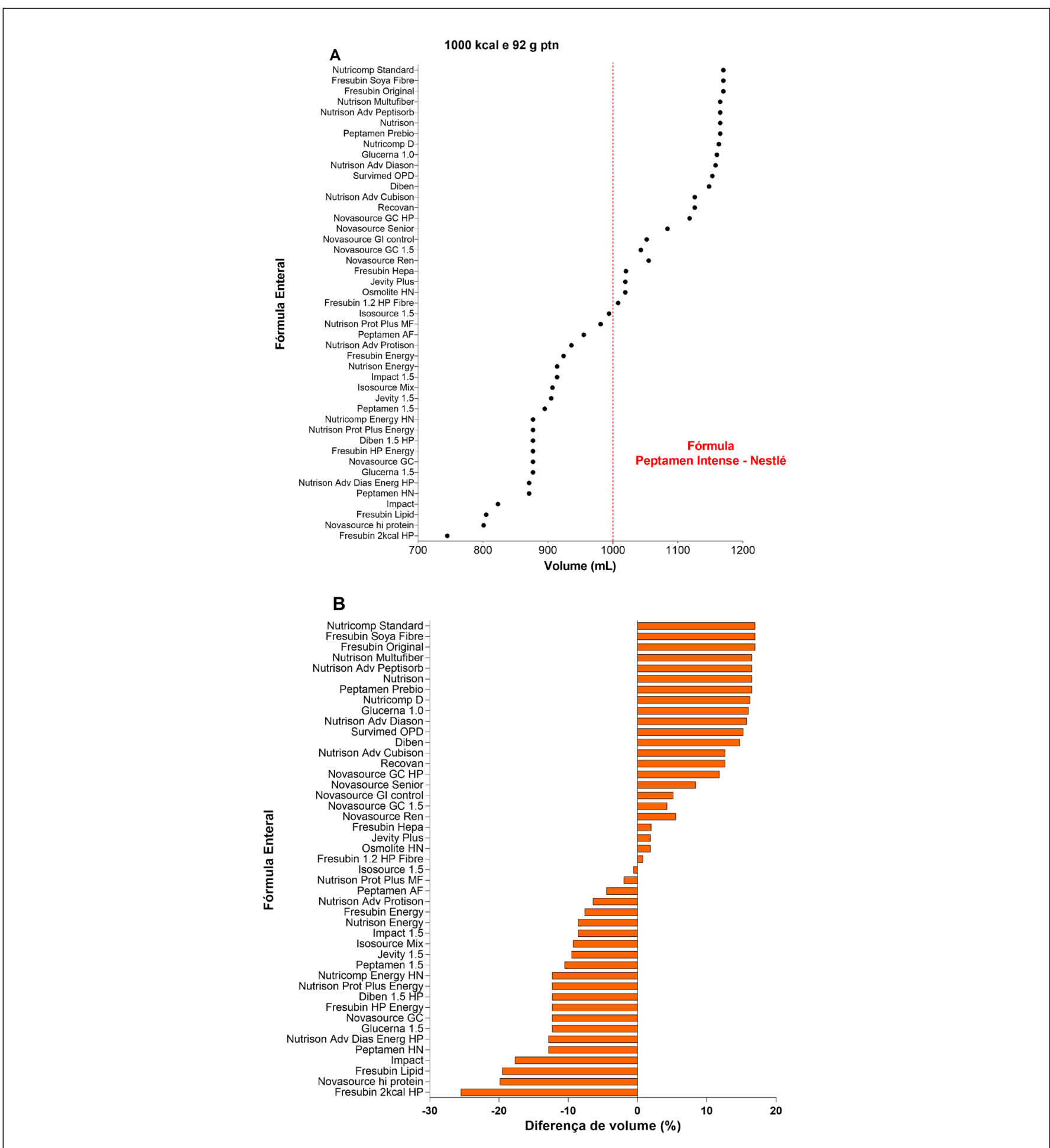

Figura 3 - Análise comparativa do volume necessário (em $\mathrm{mL}$ ) para equivalência energético-proteica entre a fórmula Peptamen Intense - Nestlé e o restante das fórmulas disponíveis no mercado acrescidas de módulo proteico. A: Volume necessário para cada fórmula isoladamente atingir $1000 \mathrm{kcal}$ e $92 \mathrm{~g}$ de proteínas. B: Diferença de volume (\%) em relação à fórmula Peptamen Intense - Nestlé. 


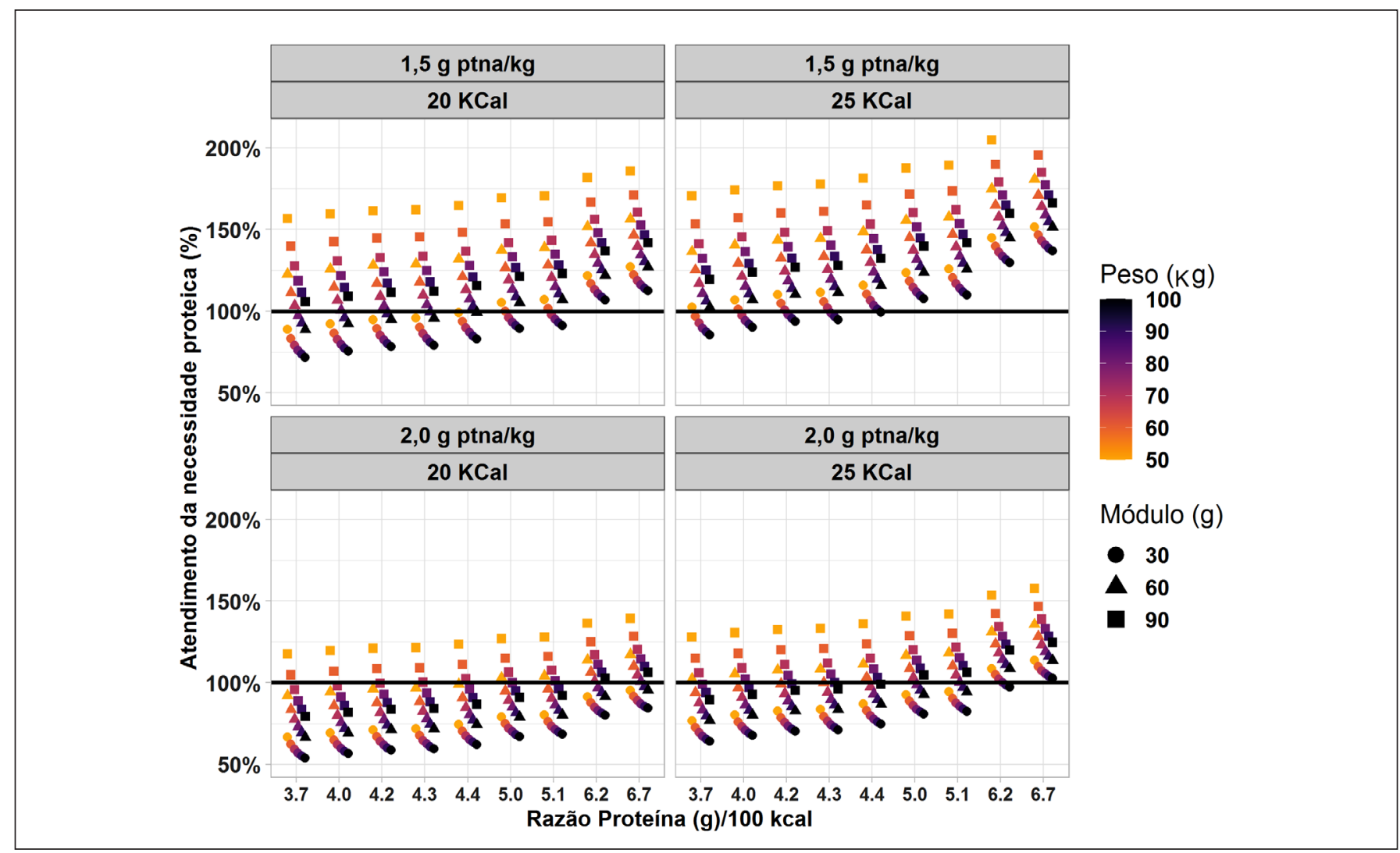

Figura 4 - Atendimento das necessidades proteicas (fórmula + módulo), em pacientes sem obesidade.

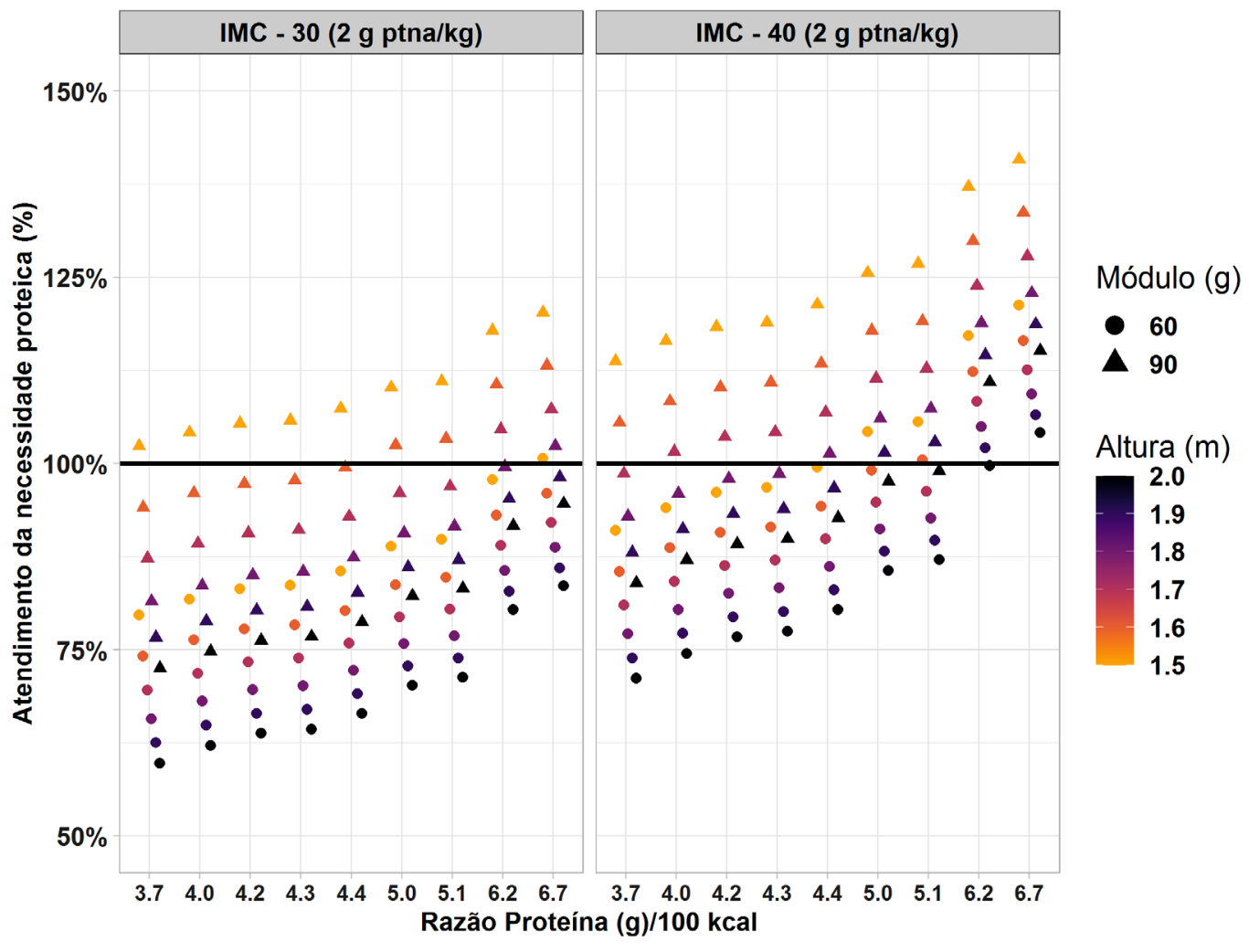

Figura 5 - Atendimento das necessidades proteicas (fórmula + módulo), em pacientes com IMC = 30 e $40 \mathrm{~kg} / \mathrm{m}^{2}$. 


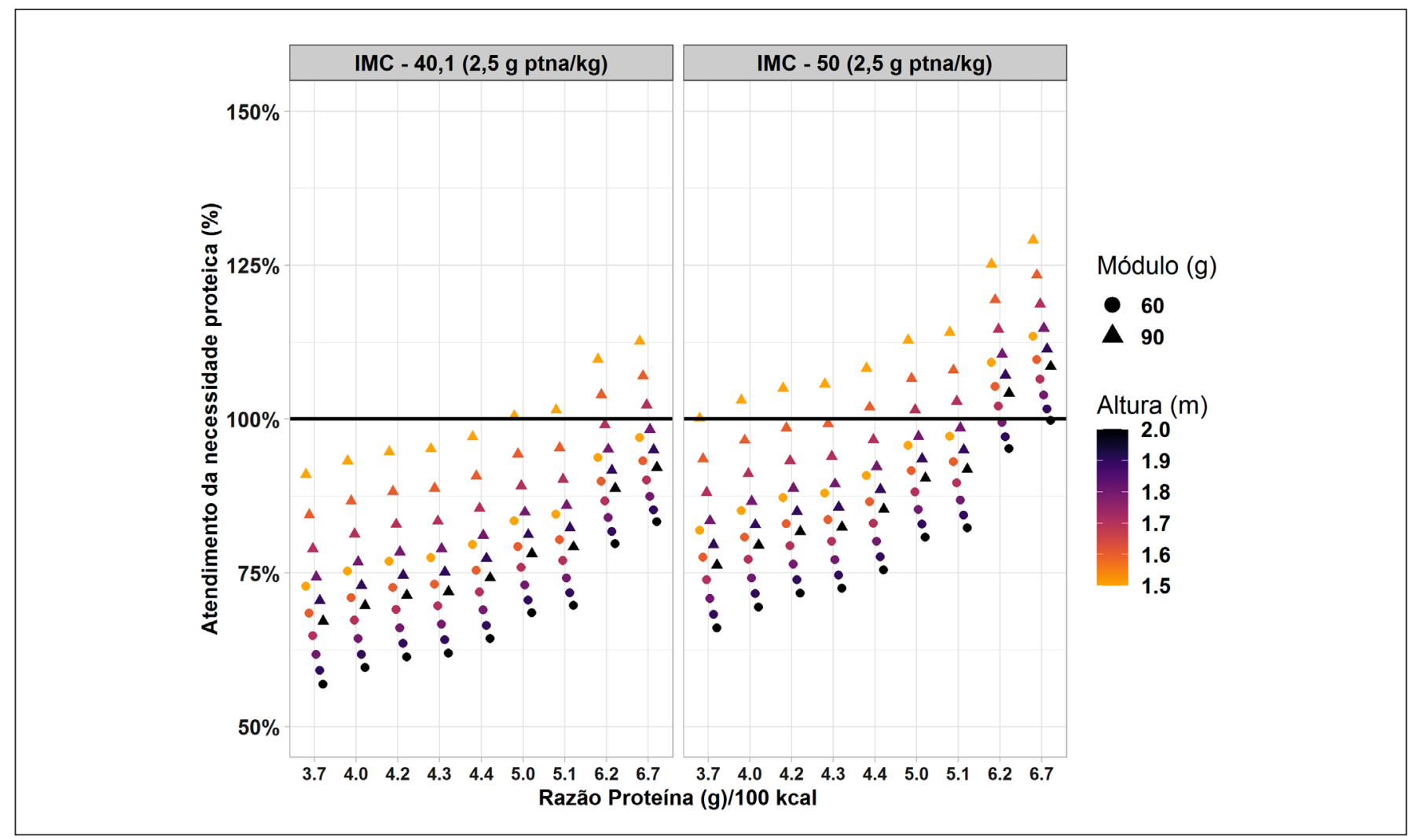

Figura 6 - Atendimento das necessidades proteicas (fórmula + módulo), em pacientes com IMC = 40,1 e $50 \mathrm{~kg} / \mathrm{m}^{2}$.

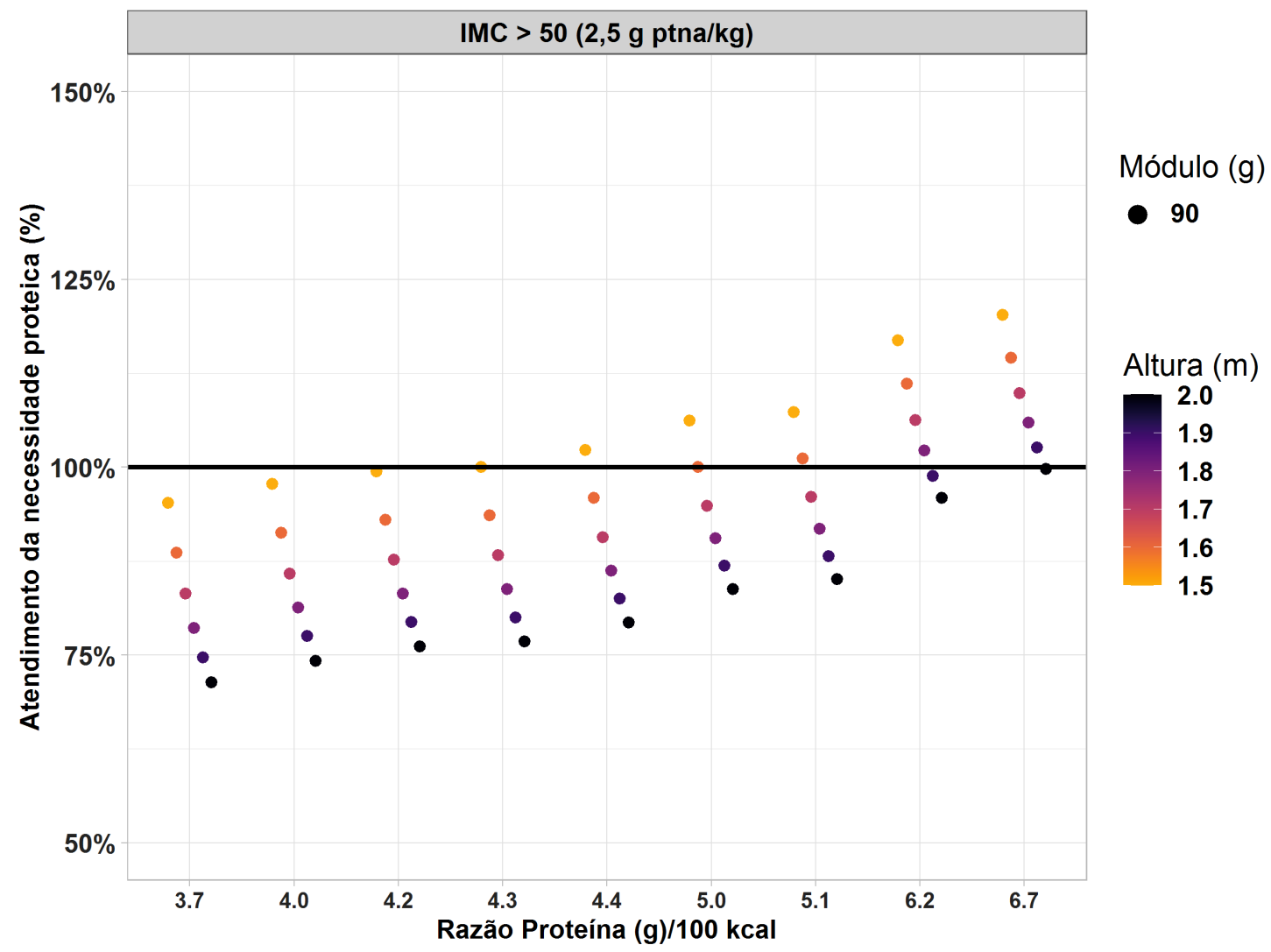

Figura 7 - Atendimento das necessidades proteicas (fórmula + módulo), em pacientes IMC maior que $50 \mathrm{~kg} / \mathrm{m}^{2}$. 


\section{DISCUSSÃO}

Ainda é escasso o número de estudos avaliando a adequação de fórmulas enterais para pacientes críticos, especialmente quando há restrição de fluidos aos doentes como na disfunção respiratória grave provocada pela COVID19. Entidades publicaram pareceres e consensos incluindo recomendações de uso de fórmulas enterais nos pacientes com a doença, porém alguns pontos são controversos. No presente estudo, observou-se que, para pacientes graves, praticamente todas as fórmulas disponíveis no mercado brasileiro não atendem às necessidades proteicas (considerando recomendações acima descritas), sem ultrapassar a necessidade energética (overfeeding). Define-se overfeeding como uma ingestão energética superior a $110 \%$ da necessidade ${ }^{10}$.

As recomendações energético-proteicas foram resumidas na Tabela 1. Apesar de estudos observacionais terem demonstrado menor mortalidade na UTI com administração mais elevada de proteína ${ }^{8,11}$, o número de ensaios randomizados controlados é pequeno e com efeitos limitados ${ }^{12,13}$, não permitindo afirmar que uma ingestão proteica muito elevada seja necessária ou mesmo segura. Revisões mais recentes recomendam 1,3 g proteína/ $\mathrm{kg}$ de peso atual/dia (ou 1,5 g proteína $/ \mathrm{kg} /$ dia se terapia nutricional enteral) para pacientes sem obesidade ${ }^{13,14}$. Ofertas $\geq 2 \mathrm{~g}$ proteína $/ \mathrm{kg} /$ dia parecem ser mais adequadas aos pacientes em uma fase mais tardia, após alta da UTI ou do hospital e sem situações especiais ${ }^{13}$.

A despeito de discrepâncias nas recomendações, atingir a necessidade proteica pode ser mais importante que atingir a necessidade energética, com menor mortalidade inclusive? Entretanto, como já descrito previamente e igualmente demonstrado neste estudo, a razão proteína $(\mathrm{g}) / 100 \mathrm{kcal}$ da maioria das fórmulas enterais disponíveis é baixa, não permitindo entregar ao paciente a recomendação proteica, sem gerar overfeeding ${ }^{15}$. Dessa forma, resta, para atingir a recomendação proteica, o emprego de módulos proteicos enterais ou soluções de aminoácidos parenterais ${ }^{6}$.

Neste estudo, encontramos que apenas uma fórmula, a Peptamen Intense - Nestlé, com 9,2 g proteína/100 kcal (proteína hidrolisada) apresenta-se capaz de prover as necessidades na maior parte das situações. No entanto, não há indicação rotineira para uso de fórmulas hidrolisadas, com estudos prévios sugerindo pior tolerância e consequente menor administração de proteína aos pacientes, se comparadas às fórmulas poliméricas ${ }^{15,16}$. De fato, em seu parecer sobre COVID-19, a American Society for Parenteral and Enteral Nutrition (ASPEN) sugere fórmula padrão polimérica'. O uso da fórmula Peptamen Intense - Nestlé é, no entanto, admissível, uma vez que não há fórmula com proteína intacta e similar razão proteína $(\mathrm{g}) / \mathrm{kcal}$.

Além da Peptamen Intense - Nestlé, outras fórmulas se mostraram capazes de suprir a necessidade energéticoproteica em pacientes sem obesidade, quando infundidas em um volume para $25 \mathrm{kcal} / \mathrm{kg} / \mathrm{dia}$, todas com razão $\geq$ $5,2 \mathrm{~g}$ proteína/100 kcal. No entanto, várias dessas fórmulas apresentam nutrientes que podem limitar o seu uso em alguns casos. As fórmulas Impact - Nestlé, Impact 1.5 - Nestlé, Novasource Prol - Nestlé, Recovan - Fresenius, Nutrison Advanced Cubison - Danone/Nutricia são enriquecidas com arginina, enquanto Novasource GC - Nestlé e Fresubin Lipid - Fresenius apresentam teor de fibra mais elevado. Já a fórmula D-6 não apresenta restrição quanto à sua composição. Até o momento, não existe conclusão sobre vantagens e mesmo segurança com o uso fibras solúveis e insolúveis em pacientes críticos, especialmente aqueles com instabilidade hemodinâmica, nos quais há risco de isquemia intestinal ${ }^{17}$. Por sua vez, há dúvidas quanto à segurança em relação ao uso de fórmulas contendo arginina em pacientes sépticos, devido ao aumento de mortalidade observado no passado ${ }^{18}$.

Como verificado, a adição de módulos de 30 a $90 \mathrm{~g}$ de proteína às várias fórmulas permite atingir a necessidade energético-proteica em diferentes situações. Obviamente, quando módulos são associados às fórmulas com maior densidade energética, o volume do conjunto (fórmula enteral + módulo) necessário para atingir as recomendações será menor. Por sua vez, quanto maior a razão proteína (g)/100 kcal da fórmula enteral, menor será a quantidade (em gramas ou $\mathrm{mL}$ ) necessária de módulo proteico para atingir a necessidade. Nesse sentido, a fórmula Fresubin 2 kcal HP, aquela com maior razão proteína $(\mathrm{g}) / 100 \mathrm{kcal}$ dentre aquelas com maior densidade energética $(2,0 \mathrm{kcal} / \mathrm{mL})$, resulta no menor volume dentre todas as comparações à Peptamen Intense Nestlé (25,5\% menor), para entregar a mesma quantidade de energia e proteína.

Assim, como sugerido previamente, é obviamente vantajosa a administração de fórmulas enterais 1,5 a $2,0 \mathrm{kcal} / \mathrm{mL}$ aos pacientes com restrição hídrica ${ }^{2}$. Além disso, pacientes graves com COVID-19, possivelmente, necessitarão de ventilação mecânica em posição prona, onde a infusão de terapia nutricional enteral pode ser desafiadora ${ }^{19}$. Existem poucos trabalhos avaliando esta situação, porém foi evidenciado que, mantendo-se a cabeceira elevada, o uso de procinético profilático (eritromicina) e a infusão da fórmula enteral até $85 \mathrm{~mL} / \mathrm{h}$, houve boa tolerância, baixo resíduo gástrico, sem aumento de complicações ${ }^{20,21}$. Conclui-se então que, pacientes em posição prona poderão receber terapia nutricional enteral, provavelmente em um volume $(\mathrm{mL} / \mathrm{h})$ não muito elevado, situação onde fórmulas com elevada densidade energética seriam vantajosas. Por fim, como verificado neste estudo, é possível entregar a necessidade energético-proteica com menor volume, por meio da adição de módulo proteico às fórmulas com $\geq 1,5 \mathrm{kcal} / \mathrm{mL}$. Ainda, ressalta-se que o custo de fórmulas enterais com alta razão proteína $(\mathrm{g}) / 100 \mathrm{kcal}$ é comumente elevado. Estudo brasileiro recente demonstrou que $35,4 \%$ do custo hospitalar total foram gastos com terapia nutricional, sendo $60 \%$ deste montante atribuídos à terapia nutricional enteral, com destaque para formulações especializadas ${ }^{22}$. Em épocas de pandemia, nas quais se espera maiores custos hospitalares, a utilização de fórmulas especializadas mais caras pode trazer impacto econômico importante à instituição hospitalar. A adição de módulo se mostra uma alternativa com menor 
custo, sem impacto significativo na qualidade assistencial, desde que executada de acordo com protocolos.

Por fim, foi levantado que o uso de módulo proteico não deveria ser estimulado durante a pandemia da COVID-19, visando à redução do risco de contaminação da equipe de enfermagem, pelo excesso de manipulação dos pacientes ${ }^{2}$. Entretanto, sabe-se que pacientes em estado grave já recebem diariamente número elevado de medicamentos por diversos tipos de acesso ${ }^{23}$. Além disso, o uso de módulos seguindo o princípio de -cluster care- não tem sido contraindicado'.

\section{CONCLUSÃO}

O presente estudo identificou que a maioria das fórmulas enterais disponíveis no mercado brasileiro não é capaz de ofertar, isoladamente, quantidade adequada de proteínas, sem resultar em overfeeding. Sabe-se que é necessário que a oferta de proteínas seja feita de forma individualizada, de acordo com a condição clínica de cada paciente, sendo que o uso de módulos proteicos pode trazer benefícios frente à recuperação dos pacientes com e sem COVID-19.

\section{REFERÊNCIAS}

1. Martindale R, Patel JJ, Taylor B, Warren M, McClave SA. Nutrition therapy in the patient with COVID-19 disease requiring ICU care [Internet]. www.nutritioncare.org. 2020 [cited 2020 Apr 10]. Available from: https://www.nutritioncare.org/uploadedFiles/Documents/Guidelines_and_Clinical_Resources/ Nutrition\%20Therapy\%20COVID-19_SCCM-ASPEN.pdf

2. Campos LF, Barreto PA, Ceniccola GD $\bar{D}$, Gonçalves RC, Matos LBN, Zambelli C, et al. Parecer BRASPEN/AMIB para o enfrentamento do COVID-19 em pacientes hospitalizados. BRASPEN. 2020;35(1):3-5.

3. Barazzoni R, Bischoff SC, Breda J, Wickramasinghe K, Krznaric $Z$, Nitzan D, et al. ESPEN expert statements and practical guidance for nutritional management of individuals with SARSCoV-2 infection. Clin Nutr. 2020;39(6):1631-8.

4. Justino S, Franzosi OS, Caruso L, Shima M, Contini LJ, Ceniccola GD. Sugestões para assistência nutricional de pacientes criticos com SARS- Cov-2 [Internet]. Associação de Medicina Intensiva Brasileira. [cited 2020 Apr 10]. Available from: https://www. amib.org.br/fileadmin/user_upload/amib/2020/marco/31/vjsASSOCIACAO_DE_MEDICINA_INTENSIVA_BRASILEIRA_ DEPARTAMENTO DE NUTRICAO DA AMIBvjs.pdf

5. McClave SA, Taylor BE, Martindale $\bar{R} G$, Warren MM, Johnson $\mathrm{DR}$, Braunschweig $\mathrm{C}$, et al. Guidelines for the provision and assessment of nutrition support therapy in the adult critically ill patient: Society of Critical Care Medicine (SCCM) and American Society for Parenteral and Enteral Nutrition (A.S.P.E.N.). JPEN J Parenter Enteral Nutr. 2016;40(2):159-211.

6. van Zanten ARH, Petit L, De Waele J, Kieft H, Wilde J, van Horssen P, et al. Very high intact-protein formula successfully provides protein intake according to nutritional recommendations in overweight critically ill patients: a double-blind randomized trial. Crit Care. 2018;22(1):156.
7. Compher C, Chittams J, Sammarco T, Nicolo M, Heyland DK. Greater protein and energy intake may be associated with improved mortality in higher risk critically ill patients: a multicenter, multinational observational study. Crit Care Med. 2017;45(2):156-63.

8. Elke G, Wang M, Weiler N, Day AG, Heyland DK. Close to recommended caloric and protein intake by enteral nutrition is associated with better clinical outcome of critically ill septic patients: secondary analysis of a large international nutrition database. Crit Care. 2014;18(1):R29.

9. Song JH, Lee HS, Kim SY, Kim EY, Jung JY, Kang YA, et al. The influence of protein provision in the early phase of intensive care on clinical outcomes for critically ill patients on mechanical ventilation. Asia Pac J Clin Nutr. 2017;26(2):234-40.

10. Singer P, Blaser AR, Berger MM, Alhazzani W, Calder PC, Casaer MP, et al. ESPEN guideline on clinical nutrition in the intensive care unit. Clin Nutr. 2019;38(1):48-79.

11. Heyland DK, Cahill N, Day AG. Optimal amount of calories for critically ill patients: depends on how you slice the cake! Crit Care Med. 2011;39(12):2619-26.

12. Allingstrup MJ, Kondrup J, Wiis J, Claudius C, Pedersen UG, Hein-Rasmussen R, et al. Early goal-directed nutrition versus standard of care in adult intensive care patients: the single-centre, randomised, outcome assessor-blinded EAT-ICU trial. Intensive Care Med. 2017;43(11):1637-47.

13. van Zanten ARH, De Waele E, Wischmeyer PE. Nutrition therapy and critical illness: practical guidance for the ICU, post-ICU, and long-term convalescence phases. Crit Care. 2019;23(1):368.

14. Lambell KJ, Tatucu-Babet OA, Chapple L-A, Gantner D, Ridley EJ. Nutrition therapy in critical illness: a review of the literature for clinicians. Crit Care. 2020;24(1):35.

15. Looijaard WGPM, Denneman N, Broens B, Girbes ARJ, Weijs PJM, Oudemans-van Straaten HM. Achieving protein targets without energy overfeeding in critically ill patients: a prospective feasibility study. Clin Nutr. 2019;38(6):2623-31.

16. Jakob SM, Bütikofer L, Berger D, Coslovsky M, Takala J. A randomized controlled pilot study to evaluate the effect of an enteral formulation designed to improve gastrointestinal tolerance in the critically ill patient-the SPIRIT trial. Crit Care. 2017;21(1):140.

17. Castro MG, Ribeiro PC, Souza IAO, Cunha HFR, Silva MHN, Rocha EEM, et al. Diretriz brasileira de terapia nutricional no paciente grave. BRASPEN J. 2018;33(supl. 1):2-36.

18. Bertolini G, Iapichino G, Radrizzani D, Facchini R, Simini B, Bruzzone $\mathrm{P}$, et al. Early enteral immunonutrition in patients with severe sepsis: results of an interim analysis of a randomized multicentre clinical trial. Intensive Care Med. 2003;29(5):834-40.

19. Baig MA. The COVID-19 intubation and ventilation pathway (CiVP); a commentary. Arch Acad Emerg Med. 2020;8(1):e37.

20. Allen K, Hoffman L. Enteral nutrition in the mechanically ventilated patient. Nutr Clin Pract. 2019;34(4):540-57.

21. Reignier J, Dimet J, Martin-Lefevre L, Bontemps F, Fiancette $\mathrm{M}$, Clementi E, et al. Before-after study of a standardized ICU protocol for early enteral feeding in patients turned in the prone position. Clin Nutr. 2010;29(2):210-6.

22. Hyeda A, Costa ÉSM. Economic analysis of costs with enteral and parenteral nutritional therapy according to disease and outcome. Einstein. 2017;15(2):192-9.

23. Moss J, Berner E, Bothe O, Rymarchuk I. Intravenous medication administration in intensive care: opportunities for technological solutions. AMIA Annu Symp Proc. 2008;495-9.

Local de realização do estudo: Divisão de Nutrologia, Departamento de Clínica Médica, Faculdade de Medicina de Ribeirão Preto, Universidade de São Paulo, Ribeirão Preto, SP, Brasil.

Conflito de interesse: Os autores declaram não haver. 